\title{
Toward a Hierarchical Mobile IPv6
}

\author{
Claude Castelluccia
}

INRIA Rhone-Alpes.

ZIRST - 655 avenue de l'Europe

38330 Montbonnot Saint-Martin

France

Claude.Castelluccia@inrialpes.fr

\begin{abstract}
The IETF Mobile IPv6 protocol provides a mobility management scheme for the Internet. Mobile IPv6 handles macro-mobility and micro-mobility identically. We believe that a hierarchical scheme that separates micro-mobility from macromobility is preferable since it would be more scalable. In this paper, we present a mobility management architecture that makes use of the IPv6 Address format hierarchy to provide an efficient and scalable architecture to manage mobility in the Internet. The proposed scheme, which is fully compatible with the IETF solution, differentiates the inter-site mobility management from the intra-site mobility management. The hosts' local mobility is handled with a local, possibly customized, protocol while the global mobility, i.e. across sites, is handled with Mobile IPv6. Our approach has two main advantages. First, the mobility of a host within a site is fully transparent to its correspondent nodes. As a result, the mobility management signaling load is minimized and some of Mobile IPv6 security issues are solved. We show that the signaling load generated by our proposal is at least $69 \%$ lower than the Mobile IPv6 one. Second, by differentiating intra-site mobility from inter-site mobility, we provide an architecture that is hierarchical, scalable, flexible and customizable; each site can deploy the intra-site mobility management scheme that is the most appropriate to its particular needs.
\end{abstract}

\section{Keywords}

Mobile host, TCP/IP networking, Mobile-IP, Mobility Management

The original version of this chapter was revised: The copyright line was incorrect. This has been corrected. The Erratum to this chapter is available at DOI: $10.1007 / 978-0-387-35388-3 \_42$ 


\section{INTRODUCTION}

Internet Mobile users require special support to maintain connectivity as they change their point-of-attachment. This support should provide performance transparency to mobile users and should be scalable. Providing performance transparency means that higher level protocols should be unaffected by addition of mobility support. Issues that may affect performance transparency are optimum routing of packets to and from mobile nodes and efficient network transition procedures (Myles, 93). The mobility support should be scalable in the sense that it should keep providing good performance to mobile users and should keep the network load low as the network grows and as the number of mobile node increases. This scalability issue is a very important one in the context of a still growing worldwide network such as the Internet.

The IETF Mobile IPv6 standard, which provides a mobility management scheme for the Internet, does not completely meet these design goals. While it provides performance transparency, we argue that Mobile IPv6 is not scalable. In Mobile IPv6, a mobile node sends a location update to each of its correspondent nodes periodically and any time it changes its point-of-attachment. The resulting signaling and processing load can become very significant as the number of mobile nodes increases. This limitation is the result of the lack of hierarchy in the mobility management procedures of Mobile IPv6. In fact, Mobile IPv6 handles macro-mobility and micro-mobility identically. Since $69 \%$ of a user's mobility is local, we believe that a hierarchical scheme that separates micro-mobility from macro-mobility is preferable.

In this paper, we present a mobility management architecture that makes use of the IPv6 Address format hierarchy to provide an efficient and scalable solution. The proposed scheme, which is fully compatible with the IETF solution, differentiates the inter-site mobility management from the intra-site mobility management. The hosts' local mobility is handled with a local, possibly customized, protocol while the global mobility, i.e. across sites, is handled with Mobile IPv6. Our approach has two main advantages over Mobile IPv6. First, the mobility of a host within a site is fully transparent to its correspondent nodes. As a result, the mobility management signaling load is minimized and some of Mobile IPv6 security issues are solved. We show that the signaling load generated by our proposal is at least $69 \%$ lower than the Mobile IPv6 one. Second, by differentiating intra-site mobility from inter-site mobility, we provide an architecture that is hierarchical, scalable, flexible and customizable. Our proposal is efficient; it provides optimum routing from and to mobile hosts and improves handoff latency. It is flexible and customizable; each site can deploy the intra-site mobility management scheme that is the most appropriate to its needs. 
This paper is structured as follows. In the next section, we introduce some terminology that is used throughout this paper. Section 3 presents the related work including the IETF Mobile IPv6 and its hierarchical derived proposals. Section 4 details our mobility management proposal. Section 5 evaluates and compares the performance of our scheme with the IETF one. Section 7 concludes our paper.

\section{TERMINOLOGY}

The following terms are used in this paper to identify the principal network entities that are of interest to our proposal. A mobile host, $M H$ is a node that may move through the Internet. A correspondent host, $\mathrm{CH}$, is a host communicating with the $\mathrm{MH}$. The network and the site of a $\mathrm{MH}$ when it is not travelling are respectively called the home network and the home site of the MH. The network and the site that a MH may visit are respectively referred as the foreign network and the foreign site of the MH. A MH's Care-of Address is the global IP address the MH acquires when visiting a foreign network. This address is topologically correct on the foreign network. A MH's Site Care-of Address is the first Care-of Address that a $\mathrm{MH}$ acquires when visiting a site. When $\mathrm{MH}$ is in its home network, it is accessible through its Home Address.

\section{RELATEDWORK}

In this Section, we present some of the mobility schemes proposed for the Internet. We start with IETF Mobile IPv6 and then describe two of its hierarchical derived approaches, which have been proposed in the context of Mobile IPv4.

The Mobile IPv6 protocol is currently being specified by the IETF IP Routing for Wireless/Mobile working group (Perkins, 96b). With Mobile IPv6, each time the mobile node moves from one subnet to another, it gets a new care-of address. It then registers its Binding (association between a mobile node's home address and its care-of address) with a router in its home subnet, requesting this router to act as the home agent for the mobile node. This router registers this binding in its Binding Cache. At this point, the router serves as a proxy for the mobile node until the mobile node's binding entry expires. The router intercepts any packets addressed to the mobile node's home address and tunnels them to the mobile's care-of address using IPv6 encapsulation. The mobile node sends also a Binding Update to its correspondent nodes, which can then send packets directly to the mobile node.

While this protocol optimizes the routing of packets to mobile hosts, it is not scalable. As the number of mobile nodes increases in the Internet, the number of Binding messages will also increase proportionally and add a significant extra load to the network. 
Caceres and al. have proposed a hierarchical mobility scheme based on Mobile IPv4 that separates three cases : local mobility, mobility within an administrative domain and global mobility in order to reduce the generated signaling load (Caceres,96). This proposal has been made in the context of Mobile IPv4 which uses foreign agents; agents that mobile hosts connect to when they visit a foreign network. (Caceres,96) defines a hierarchy of foreign agents. In this proposal, each subnet that a mobile node could visit has one or more subnet foreign agents, which manage local mobility. On top of those subnet foreign agents, a domain foreign agent manages mobility across the different subnets of an administrative domain. The mobile node's home agent only keeps track of the movement of the mobile node across administrative domain boundaries. As a result, the mobile node's motion within an administrative domain is transparent to the home agent and its correspondent nodes. The hierarchical architecture of this scheme is very interesting but strongly relies on the deployment of foreign agents, which makes it incompatible with the MobileIPv6 protocol.

In the scheme, proposed by Balakrisnan et al. (Balakrisnam,95), packets destined for a mobile node are delivered to the mobile node's home agent using the IETF Mobile IPv4 and are then multicast to multiple base stations in close vicinity of the mobile node. While this approach is hierarchical, we believe that this solution is not very efficient and scalable. In fact, packets destined for a mobile have to transit through the home agent which can be distant from the mobile node's current location. This has the effect of increasing packet delivery latency, handoff latency and the Internet load.

\section{AHIERARCHICAL MOBILITYMANAGEMENT ARCHIIECTURE}

Mobile IPv6 handles local mobility of a host (i.e. within a site or a network) the same way as it handles global mobility (inter-site or inter-network mobility). In fact, in Mobile IP, a mobile user sends location updates to its home agent and its correspondent nodes each time it changes its point-of-attachment regardless of the locality and amplitude of its movement. As a consequence, the same level of signaling load is introduced in the Internet independently of the user's mobility pattern.

We argue that this approach is not scalable and that a hierarchical solution is more appropriate to the Internet. We believe that a user's mobility within a site or a network should be managed locally and transparently to its correspondent nodes. Using such a hierarchical approach has at least two advantages. First, it improves handoff performance, since local handoffs are performed locally. This increases the handoff speed and minimizes the loss of packets that can occur during the transition phase. Second, it significantly reduces the mobility management signaling load on the Internet since the signaling load corresponding to local moves do not cross the whole Internet but is confined to the site or the network. This 
hierarchy is furthermore motivated by the significant geographic locality in user mobility patterns. As shown in (Kirk,95), most of a user's mobility is local. According to this study, $69 \%$ of a user's mobility is within its home site (within its building and campus) and $70 \%$ of all professionals can be classified as mobile. It is therefore important to design a hierarchical mobility management architecture that optimizes local mobility.

We propose a hierarchical architecture that separates local mobility (within a site) from global mobility. Our architecture is hierarchical in two points. First, it separates the local mobility management from the global one. Local handoffs are managed locally and transparently to mobile's correspondent hosts. Second, it clearly separates the protocols managing local mobility from the protocols managing global mobility. In fact, while the hierarchy in the mobility management operations could be performed by the same protocol, we propose to use two different protocols. As illustrated by Figure 1, we define the concepts of MISP (Mobility Internal Site Protocol), that manages mobility within a site, and of MESP (Mobility External Site Protocol), that manages mobility between sites. The concept of site is quite general. We use the definition of site as it is given in (Hinden,98). A site is a set of networks belonging to the same administrative entity, such as a company or an access provider. Any two hosts of a site must be able to exchange packets without the support of the Internet backbone. A site is connected to the rest of the Internet via one or several interconnection routers. The approach that we propose provides more flexibility to the sites that can deploy the MISP the most appropriate to their needs. A large site can, for example, use a hierarchical mobility management protocol, and add an extra level of hierarchy to the global architecture.

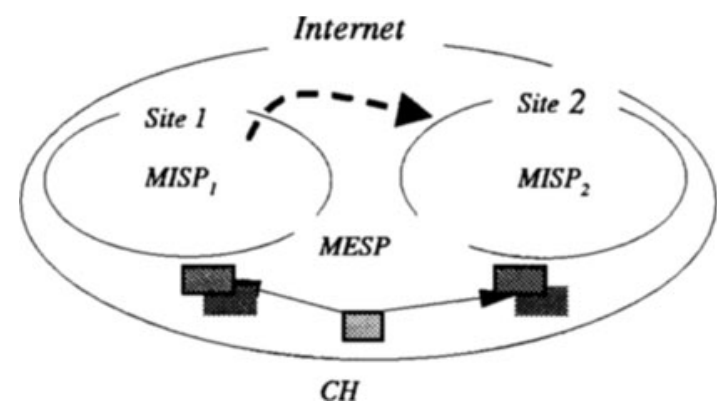

Figure 1 MISPs and MESP 


\subsection{Inter-site Mobility Management Issues}

The Inter-site Mobility Management protocol manages the mobility of hosts between sites. This protocol has to be global to the whole Internet. We propose to use the Mobile IPv6 protocol since it is the current IETF solution and we believe that it is an efficient solution to manage macro-mobility. As with the "regular" Mobile IPv6, a mobile host requires the service of a home agent in its home network. This HA intercepts packets addressed to the $\mathrm{MH}$ and forwards them toward the MH's current Care-of Address.

When a mobile host gets into a new site, it obtains a Care-of Address, and communicates it to its home agent and possibly to its correspondent nodes via the emission of a Binding Update composed of its Home Address and its Care-of Address. Thereafter, and as long as the mobile host stays within this site, this Careof Address, that we call the Site Care-of Address, is used in all Binding Updates sent to the Home Agent and Correspondent Hosts. Note that this Site Care-of Address is used in the Binding Updates even if the mobile host moves within the site and gets new Care-of Addresses.

Upon reception of a binding, the $\mathrm{HA}$ and $\mathrm{CH}$ update their binding list and use the Site Care-of Address specified in the BU to communicate with the $\mathrm{MH}$, in conformance with the Mobile IP protocol specification (Perkins, 96b).

\subsection{Intra-site Mobility Management Issues}

The Intra-site Mobility Management protocol manages the mobility of hosts within a site. As opposed to the MESP, the MISP can differ from sites to sites. They can be customized to each site's needs. For example, a large site may deploy a hierarchical MISP while a smaller one may use Mobile IPv6. In the next section, we describe a Mobile IPv6-based MISP. This solution results in a 2 level-Mobile IPv6 protocol: one level manages macro-mobility (MESP) and the other one manages micro-mobility (MISP).

\subsubsection{Mobile IP-based MISP}

When a mobile host moves within a site and changes its point-of attachment, it gets a new Care-of address, $\mathrm{CoA}_{2}$, and sends a Binding Update, composed of $\mathrm{COA}_{2}$ and its Site Care-of Address, $\mathrm{CoA}_{S}$, to all of the site interconnection routers ${ }^{1}$ of the site and a Binding Update composed of its Home Address and its current Care-of Address, $\mathrm{CoA}_{2}$, to its local correspondent nodes. Each interconnection router of the

\footnotetext{
' All the interconnection routers of a site could be made accessible via the use of a well-know IPv6 multicast address or via a multicast address communicated to the MHs by the Neighbor Discovery protocol.
} 
site maintains a Binding list with one entry $\left(\mathrm{CoA}, \mathrm{CoA}_{S}\right)$ for each mobile host currently roaming in the site.

When a packet addressed to a mobile host arrives at one of the site's interconnection router, this router searches into its Binding list for an entry whose Site Care-of Address field matches the destination address of the incoming packet ${ }^{2}$. (1) If no entry is found, the packet is routed normally with in the site. (2) If an entry is found, the router tunnels the packets to the current (local) Care-of address of the mobile host as specified in Mobile IPv6.

When a host sends packets to a mobile host that is located within its site, it first uses the mobile home address and then switches to the mobile host's Care-of Address as soon as it receives a Binding Update. As a result, if the site is the home site of the mobile host, the first packet is intercepted by the mobile host's home agent and tunneled to its current site address. If the site is not the home site of the mobile host, the first packet is intercepted by one of the interconnection routers of the site and then tunneled to the mobile host's site address.

\subsubsection{Others MISPs}

Others MISPs could be deployed. For example, the Sony VIP (Teraoka,92) and the Columbia MHP (Bhawat,95), that were designed for small networks, could be good candidates for MISPs. The PIM-based mobility management scheme presented in (Castelluccia,98) could be used for larger sites. A GSE-based approach (O'dell,98) could also be considered. In this approach, the site interconnection routers would dynamically replace the destination address of the packet addressed to a mobile host home address or Care-Of Address with the current Site Address. While this solution prevents from encapsulating packets and makes better use of the local resource, it introduces some security and identification problems.

\subsubsection{MISPs Compatibility Issues}

An important consideration in our architecture is the MISP compatibility. In fact, it is important, for extensibility reasons, that a mobile host is able to use the different MISPs without having to understand all of them. Therefore, the operations performed by the mobile hosts in the different MISPs have to be standardized.

We propose that the mobile host operations be limited to the emission of Binding Updates to one or several special addresses. These addresses, that can change from MISP to MISP, could be communicated to the mobile host through the IPv6

\footnotetext{
${ }^{2}$ Maintaining these per-mobile host entries is not necessarily a scalability limitation since data structures exist that allow routers to handle long lists of entries efficiently (Sklower,93).
} 
Neighbor Discovery mechanism. For example in the Mobile IP-based MISP this address is the multicast address of the site interconnection routers .

\subsection{The IPv6 Mobility bit}

The site interconnection routers play a central role in our architecture. In fact, they filter all incoming packets to demultiplex packets addressed to mobile hosts from those addressed to fixed hosts. This operation can be expensive if the routers must compare the destination address of all incoming packets against the list of mobile hosts roaming within the site. To minimize this cost, we propose the definition of a mobility bit within the IPv6 Addresses to help routers to efficiently demultiplex packets addressed to mobile hosts from those addressed to fixed host.

The IPng Working Group has defined an global address format for IPv6, the Aggregatable Global Address Format (Hinden,98). This address, that is presented in Figure 2, is structured into a three level hierarchy : (1) Public topology (48 bits), (2) Site topology (16 bits) and (3) Interface Identifier (64 bits). The public topology is the collection of providers and exchange points. The Site topology is local to a specific site or organization. It is used by an individual organization to create its own local addressing hierarchy and to identify subnets. Interface identifiers identify interfaces on links.

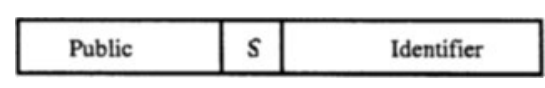

Figure2 IPv6 Aggregatable Global Unicast Address Format

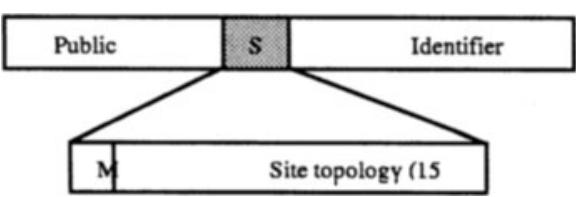

Figure 3 Modified IPv6 Unicast Address

For performance concerns, we propose to define a Mobility bit within the Site topology field of the IPv6 address format (see Figure 3). This bit, which is only meaningful within a site, is used by the site interconnection routers to demultiplex packets addressed to a mobile host from the packets addressed to a fixed host efficiently. The mobility bit of a host is set to 1 in mobile hosts' addresses and set to 0 in fixed hosts' addresses. By examining this bit, the site interconnection routers can instantly know if the incoming packet should be routed internally by the standard routing protocol or the local MISP. As a result, the packets addressed to fixed hosts do not suffer from the routers' MISP processing. The mobility bit is not a requirement. It is just a suggestion to speed packets' processing at routers. Note that the mobility bit does not require to be deployed in every sites and does not affect the routing of packets on the backbone since it is only meaningful and used within a site. 


\section{COMPARISONANDEVALUATION}

In this section we compare the performance of our proposal and of Mobile IPv6. When comparing the performance of different mobility management schemes, several factors have to be taken into consideration. Among these factors, three are particularly important (Myles,93): (1) The scalability property of the schemes, i.e. how do the schemes behave as the network grows and the number of mobile hosts increases. (2) The routing performance of the schemes, i.e. what is the extra latency introduced by each of the schemes. (3) The transition performance of the schemes, i.e. how fast are the transition phases performed.

\subsection{Routing and Transition Performance}

The routing and transition performances of both schemes are quite similar. The routing is optimum, packets follow the shortest path from the correspondent nodes to the mobile host, and handoffs are performed locally in both proposals. In fact, in our proposal, local handoffs are managed within the site. In Mobile IPv6, while location updates have to cross the whole Internet to reach the mobile host correspondent nodes, a mechanism is provided to smooth out transitions. After switching to a new default router, a mobile node may send a Binding Update to its previous default router, asking him to redirect all incoming packets to its new Care-of Address.

\subsection{Scalability Performance}

The main performance difference between the compared approaches resides in their scalability property. The scalability property of a protocol can be evaluated in terms of its overhead growth on the Internet with the size of the Internet, the number of mobile hosts and the number of correspondent nodes. This overhead can be evaluated by comparing, for each proposal, their memory requirements and their signaling load, i.e. the bandwidth used by the control messages, such as the Binding Updates.

\subsubsection{Memory Requirement}

We evaluate, in this Section, the memory requirement of each proposal.

Mobile IPv6 requires that (1) each mobile node maintains a list of its correspondent nodes and (2) each correspondent node maintains a binding per mobile host it is communicating with. The corresponding memory requirement,

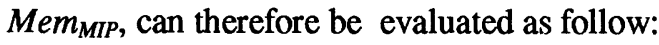

Mem $_{M T P}=2 \times \# M H \times \# C H \times$ Size $_{\text {entrv, }}$ 
Where $\# M H$ is the average number of mobile hosts on the Internet, \#CH is the average number of correspondent hosts of each mobile host and Size $_{\text {entrv, }}$ the size of the binding that a $\mathrm{CH}$ must maintain per $\mathrm{MH}$, and a $\mathrm{MH}$ must maintain per $\mathrm{CH}$.

Our hierarchical proposal requires that (1) each interconnection router of a site maintains a binding per mobile currently visiting the site, (2) each mobile node maintains a list of its correspondent nodes and (3) each correspondent node maintains a binding per mobile host, it is communicating with, that is not roaming within its home site. The corresponding memory requirement of our approach, Mem $_{\text {HмIP }}$, can therefore be evaluated as follows:

Mem $_{\text {HMIP }}=[(1+\gamma) \times \# C H+\#$ Routers $] \times \# M H \times$ Size entry

Where \#Routers is the average number of interconnection routers of a site in the Internet and $\gamma$, the percentage of the non-local mobility. According to [Kirb95], $\gamma=0.31$ therefore,

Mem $_{H M I P}=[(1.31) \times \# C H+\#$ Routers $] \times \# M H \times$ Size $_{\text {entry }}$

The gain (or loss) of our approach over the Mobile IP approach is defined as:

$G_{M e m, A V}=\left(M_{e m} m_{M P}-M_{e m} m_{A B A}\right) / M^{2} m_{M I P}$ Or,

$G_{\text {Mem,AV }}=0.345-\#$ Routers/ $(2 \times \# C H)$,

This gain is displayed in Figure 4 as a fonction of (\#CH/\#Routers).

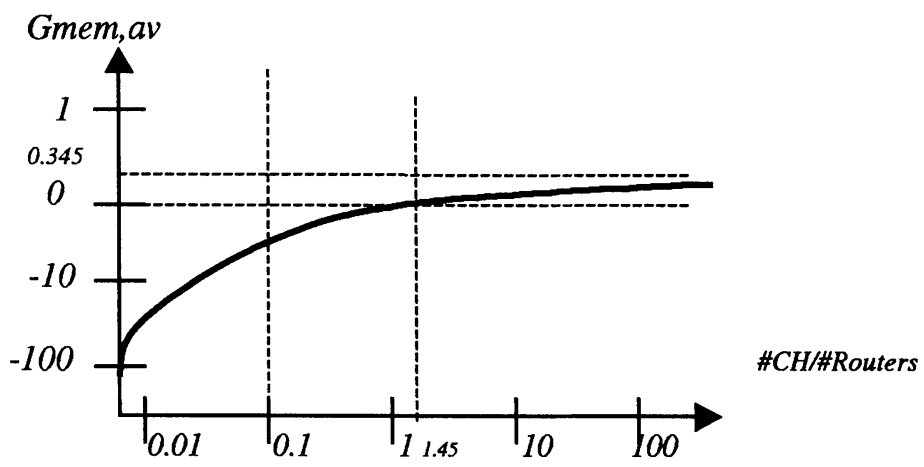

Figure 4 Memory Gain

These results show that our approach's memory requirement is lower than the Mobile IP one if \#CH is larger than a threshold, $T$, equal to 1.45X \#Routers.

\subsubsection{Signaling Load Overhead on the Internet (Backbone)}

Both mobility management proposals use Binding Updates to set up states in the routers and/or the end-hosts. This signaling has a cost in terms of bandwidth 
utilization on the Internet. In this section, we compare the signaling load introduced by both proposals on the Internet (backbone). We evaluate, for each of these schemes, the aggregated signaling load bandwidth consumed on the Internet. This aggregated bandwidth is independent of the number of nodes that the Binding Updates have to cross until their destinations, but rather corresponds to the signaling bandwidth on one link. To simplify, we do not consider the Acknowledgement messages that can sometimes be sent in response of Binding Updates. We do not compare the local signaling load because they are comparable for both schemes and because we argue that local resource is not the most critical. In this evaluation, we differentiate three types of mobility: the local mobility of a host within its home site, the local mobility of a host within a foreign site, and the inter-site mobility of a host. We then evaluate the average signaling load over these three mobility patterns.

\section{Binding Update Emission Frequency}

The signaling load of a scheme depends directly on the Binding Update Emission Frequency. According to (Perkins, 96b), a Binding Update is sent periodically to refresh the corresponding cache entries, and anytime a mobile host change its point-of attachment. The emission frequency of a Binding Update, freq, is therefore dependant on the mobility pattern of a host, rreq $_{M O V}$ and the refresh frequency, freq $q_{R E F}$. It is defined as follows:

If freq $_{\mathrm{REF}}>$ freq $_{\mathrm{Mov}}$

$$
\begin{aligned}
& \text { Freq }=a \times \text { freq }_{\text {MOV }} \text { if freq } q_{R E F}>\text { freq } q_{M O V} \\
& \text { Freq }=a^{\prime} x \text { freq } q_{\text {REF }} \quad \text { if freq } q_{M O V}>\text { freq }_{\text {REF }}
\end{aligned}
$$

With

$$
a=\lceil\text { freqREF } / \text { freqMov }\rceil \quad a^{\prime}=\lceil\text { freqMov } / \text { freqREF }\rceil
$$

\section{Local Mobility within the Home Site}

When a mobile host, using Mobile IPv6, is moving within its Home site, it sends a Binding Update to each of its correspondent nodes and to its home agent at a frequency of freq. If our hierarchical proposal is used, no Binding has to be sent at all.

As a result, the signaling bandwidths respectively generated by Mobile IP,

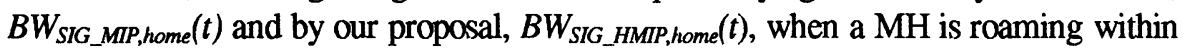
its home site, are defined as follows:

$$
\begin{aligned}
B W_{S_{I G} M I P, h o m e}(t) & =\text { Size }_{B U} x \text { freq } x \# C H, \\
B W_{\text {SIG_HMIP,home }}(t) & =0 .
\end{aligned}
$$


where Size $_{B U}{ }^{3}$ is the size of a Binding Update and \#CH is the number of correspondent hosts that are not in the home site.

\section{Local Mobility within a Foreign Site}

When a mobile host, using Mobile IPv6, is moving within a foreign site, it sends a Binding Update to each of its correspondent nodes and to its home agent at a frequency equal to freq. If our proposal is used, the mobile host only sends a Binding Update to each of its correspondent nodes and to its home agent at a frequency equal to the refresh frequency, freq $_{\mathrm{REF}}{ }^{4}$. As a result, $\mathrm{BW}_{\mathrm{SIG} \_\mathrm{MIP}, \text { foreign }}$ and $\mathrm{BW}_{\mathrm{SIG} \_\mathrm{HMIP,foreign}}$ are defined as follows:

$$
\begin{aligned}
& B W_{S I G \_M I P \text { foreign }}=\text { Size }_{B U} x \text { freq } x(\# C H+1) \\
& B W_{S I G \_H M I P \text { foreign }}=\text { Size }_{B U} x \text { fre } q_{R E F} x(\# C H+1)
\end{aligned}
$$

\section{Inter-Site Mobility}

The signaling bandwidth introduced on the Internet when a mobile node is transiting from one site to another is the same in both schemes. For each of these schemes, the mobile sends a Binding Update to its home agent, distant correspondent hosts and to the correspondent hosts that were in its previous site. Therefore, $B W_{S I G, \text { transit }}$ is defined as follows:

$$
B W_{S I G, \text { transit }}=\operatorname{Size}_{B U} x(\# C H+\# c h+1)
$$

Where \#ch is the number of correspondent hosts that are located in the previous site.

\section{Analysis of the Results}

In this section, we evaluate, for each of the mobility pattern, the gain achieved by our proposal over Mobile IPv6, $G$. We note $G_{\text {home }}$ the gain when the host is moving within its home site, $G_{f o r e i g n}$ the gain when the host is moving within a foreign site, and $G_{\text {transit }}$ the gain when the host is transiting from one site to another. $G_{y}$ (with $Y=$ home or foreign), and $G_{\text {transit }}$ are defined as follows:

$$
\begin{aligned}
& G_{Y}=\left(B W_{\text {SIG_MIP,Y }}-B W_{\text {SIG_HMIP,Y }}\right) / B W_{\text {SIG_MIP,Y }} \\
& \left.G_{\text {transit }}=\left(B W_{S I G \_M I P, t r a n s i t}-B W_{S I G \_H M I P, t r a n s i t}\right)\right) / B W_{S I G \_M I P, t r a n s i t}
\end{aligned}
$$

We also evaluate the average gains, $G_{A V}$ over the three mobility patterns, by using the result established in (Kirk,95) that $69 \%$ of a host's mobility is local. $G_{A V}$ is defined as follows:

\footnotetext{
${ }^{3}$ The size of a Binding Update is equal to the size of an IPv6 header ( 40 bytes) + the size of a Binding Update Extension Header ( 28 bytes), so 68 bytes. A Binding Update can however be smaller if it is sends with some payload.

${ }^{4}$ The Binding Updates sent to the local correspondent hosts do not cross the Internet
} 
$G_{A V}=0.69 \times G_{\text {home }}+0.31 \times\left(\alpha \times G_{\text {foreign }}+\beta \times G_{\text {transit }}\right)$

where $\alpha+\beta=1, \alpha=(N-1) / N$ and $\beta=1 / N, N$ being the average number of different points-of attachment of a mobile host within a site.

$\alpha$ and $\beta$ characterizes the mobility pattern of a user outside of its home site. $\alpha$ defines the intra-site versus inter-site moves ratio of the mobile hosts. A large $\beta$ means that the user is frequently changing sites. A large $\alpha$ means that the user is amainly roaming within a site and barely changes sites. For example, a $\alpha$ of 0.9 means that the mobile host changes, in average, 10 times its point-of attachment within a site before moving to another site.

$\alpha$ and $\beta$ can be written as functions of $T$ and freq $q_{M O V}$ :

$\beta=1 /\left(\right.$ T $x$ freq $\left.q_{\text {Mov }}\right)$

The achieved gains computed with the previous results are presented in the following table.

\begin{tabular}{|l|c|}
\hline$G_{\text {home }}$ & 1.0 \\
\hline$G_{\text {foreign }}$ & (freq-freq $\mathrm{REF}_{\mathrm{R}}$ )/freq ) \\
\hline$G_{\text {transit }}$ & 0 \\
\hline Average Gain & $\begin{array}{l}0.69+0.31 . \alpha \\
\mathrm{x}\left(\text { freq-freq }_{\mathrm{REF}}\right) / \mathrm{freq}\end{array}$ \\
\hline
\end{tabular}

Table 1 Signaling Load Gains

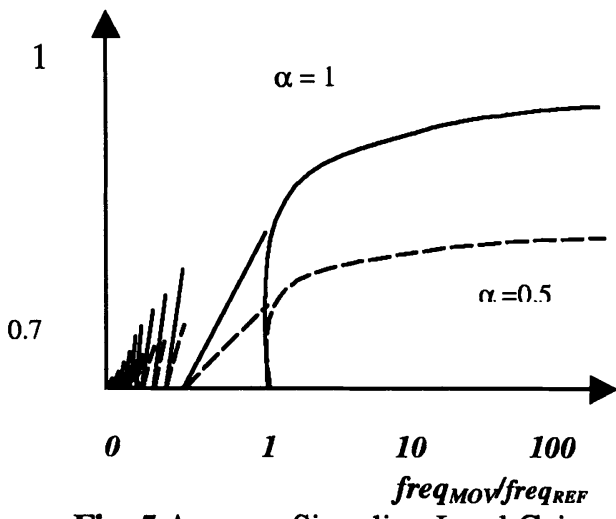

Fig. 5 Average Signaling Load Gain

These results show that our proposal's average signaling load on the Internet is at least $69 \%$ lower than the signaling load generated by Mobile IP.

According to equations (6) and (7), $f=\left(\right.$ freq-freq $\left.q_{R E F}\right) / f r e q$ is defined as follows:

$f=(a-1) / a \quad$ if freq $q_{M o v} /$ freq $q_{R E F}$

$f=\left(a^{\prime}-\right.$ freq $\left._{R E F} / f r e q_{M O V}\right) / a^{\prime}$ if freq $_{R E F} /$ freq $q_{M O V}$

Figure 5 shows the average gain $\mathrm{G}_{\mathrm{AV}}$ as a function of fre $q_{M O V} /$ fre $q_{R E F}$ for $\alpha$ equal to $1 / 2$ and 1 . This figure shows that the gain of our approach over Mobile IP is always larger than $69 \%$ and gets larger when a mobile host has a high mobility frequency, freq $q_{M O V}$, and is mainly roaming within a site ( $\alpha$ is close to 1.0 ). The peaks that 
appear when freq $_{M O V}$ is smaller than freq $q_{R E F}$ exhibit a synchronization problem of Mobile IPv6. In fact, when freq $q_{M O V}$ is smaller and is not a multiple of freq $q_{R E F} 2$ consecutive Binding Updates are sent: one to refresh the cache entries of the correspondent hosts followed by the Binding Update sent when the mobile host changes its point-of attachment. This problem does not exist with our approach since the second binding is not sent on the Internet but is confined inside the site.

\section{DISCUSSIONS ANDCONALUSIONS}

This paper presents a mobility management architecture for the Internet that is hierarchical and flexible. The proposed scheme, which is fully compatible with the IETF solution, differentiates the inter-site mobility management from the intra-site mobility management. The hosts' local mobility is handled by a local, possibly customized, protocol while the global mobility, i.e. across sites, is handled by Mobile IPv6.

When a mobile host is roaming within its home site its mobility is fully hidden from its external correspondent nodes that see the mobile host as a regular fixed host. This property could be achieved with "regular" Mobile IPv6 by using the triangular routing mode (i.e. the mobile host does not send any binding update to its correspondent nodes). However in this case, and as opposed to our proposal, all packets addressed to a mobile host are first delivered to its Home Agent and then forwarded to the current mobile host's care-of address. These indirections can drastically increase the latency and increase the network load if the home site of the mobile host is large and the mobile host is far away from its home agent. Our proposal proposes to use several home agents per mobile host. One which is located on the mobile host's home subnet, as in the regular Mobile IPv6 protocol, to handle local communications (between the mobile host and correspondent hosts of the site) and the others located on the site interconnection' routers to handle external communications (between the mobile host and correspondent hosts outside the mobile host's home site).

When a mobile host is roaming within a foreign site, its local mobility (i.e. within this site), is hidden from its correspondent hosts. These hosts are aware that the mobile host is visiting the foreign site but are unaware of its local moves. This level hierarchy is not provided with the current IETF Mobile IPv6 proposal, which requires that a correspondent host be aware of all mobile host's moves. Note that Mobile IPv4, which is the protocol that manages mobility in IPv4, defines the concept of Foreign Agents. A foreign agent is a agent a mobile host may register with when it is visiting a foreign network. Packets addressed to the mobile host are then delivered to its foreign agent and forwarded to the mobile host. Foreign agents have originally been defined to limit the constraint of mobility on the short 
IPv4 address space ${ }^{5}$, but (Caceres,96) shows that they can also be very useful in defining a hierarchical mobility management scheme. However foreign agents have not be maintained in Mobile IPv6, since IPv6 provides a much larger address space than IPv4. We argue that foreign agents should be reconsidered and adapted to Mobile IPv6 to define an hierarchical scheme. This paper proposes to deploy Mobile IPv6 foreign agents in the site interconnection's routers to hide mobile host's local moves from their correspondent nodes. These agents provide functions that are very similar than the home agents ones.

As shown in this paper, using a hierarchical mobility management scheme reduces the mobility management signaling load. In fact, we show that the signaling load generated by our proposal is more than $69 \%$ lower than the Mobile IPv6 one. When a mobile host is roaming within its home site, no binding update has to be sent over the Internet. Beside from the Internet resource saving, eliminating the signaling has several other advantages. First, it reduces the risk of attacks and tracking of a mobile host. It also eliminates the need of authentication and encryption of the Binding Updates and the associated difficult issue of the keys distribution over the Internet. Two, eliminating the signaling allows to provide mobility management support to sites which are connected to the Internet with a unidirectional and/or an asymmetrical link, such as a satellite link. Three, eliminating the signaling load is important for scalability reasons. Mobile IPv6 requires that each host maintains one entry per mobile host it is communicating with. This requirement can be overwhelming for big servers, such as Web servers, that must maintain one entries for each of its mobile clients. By handing locally the moves of mobile hosts within their home site, we reduce the number of mobile hosts on the Internet and consequently the number of entries that each correspondent host should maintain.

\section{REFERENCES}

Hari Balakrisnan, Srinivasan Seshan and Randy H.Katz (1995). Improving Reliable Transport and Handoff Performance in Cellular Wireless Networks. Proceedings of the first Annual International Conference on Mobile Computing and Networking (Mobicom'95), Berkeley, California, USA.

Pravin Bhagwat, Satish Tripathi and Charles Perkins (1995). Network Layer Mobility: An Architecture and Survey. Technical report CS-TR-3570, University of Maryland.

\footnotetext{
${ }^{5}$ In Mobile IPv4, a mobile host does not get a new care-of address but borrows the address of its foreign agent
} 
Ramón Cáceres, Venkata N. Padmanabhan (1996). Fast and Scalable Handoffs for Wireless Internetworks. In Proceedings of the Second Annual International Conference on Mobile Computing and Networking, Rye, New York, USA.

Claude Castelluccia (1998). A Hierarchical Mobilility Management Scheme for IPv6. Proceedings of the Third IEEE Symposium on Computers and Communications, Athens, Greece.

Mike O'Dell. GSE (1997) - An Alternate Addressing Architecture for IPv6. Draftietf-ipngwg-gseaddr-00.txt.

R. Hinden, M O'Dell and S. Deering (1998). An Aggregatable Global Unicast Address Format. Internet Draft.

G. Kirby (1995). Locating the User, Communication International.

Andrew Myles and David Skellern (1993), Comparing Four IP Based Mobile node Protocols. In Proceedings of the $4^{\text {th }}$ Joint European Networking Conference, Thondheim, Norway, pp. 191-196.

Charles E. Perkins, editor (1995). IP Mobility Support, Request For Comment, RFC2002.

Charles E. Perkins and David B. Johnson (1996), Mobility Support in IPv6. In Proceedings of the Second Annual International Conference on Mobile Computing and Networking, Rye, New York, USA.

K. Sklower (1991), A Tree-Based Packet Routing Table for Berkeley Unix. In Proceedings of the 1991 Winter USENIX Technical Conference.

Fumio Teraoka et al. (1992), Design Implementation and Evaluation of Virtual Protocol. In Proceedings of the $12^{\text {th }}$ International Conference On Distributed Computing Systems, pp.170-177.

\section{BIOGRAPHY}

Claude Castelluccia is a research scientist at INRIA Rhone-Alpes, France. He holds a MSEE from Florida Atlantic University, USA, and a PhD from INRIA Sophia-Antipolis, France. His research interests includes mobile internetworking, protocol design and signal processing. 